\title{
Inhibition of Hypertension and Organ Changes under Long-Term Administration of Anti- Hypertensive Drugs to SHR
}

\author{
Takahisa Uchiyama, Nagao Kajiwara, Yoshiko Kobayashi, \\ Akira Murakami, Jun Hashida, Takashi Kita, Masahiko Sato, \\ Masashi Ono, Yasuo Izeki, Tateo Ter, Hiroshi Terashima, \\ Nobuaki Mrshina, Toshio Kushiro, Yozo Fujibayashi, Akie Nakamura, \\ Michinobu Hatano, and Kazumi Sato*
}

This experiment aims to know how much organ damages could be inhibited, when anti-hypertensive drugs are administered for a long term to SHR of prehypertensive stage.

Method:

Male SHRs, 5 weeks of age, were orally administered fusaric acid (DBH inhibitor) $150 \mathrm{mg} / \mathrm{Kg} /$ day, hydralazine $30 \mathrm{mg} / \mathrm{Kg} /$ day, and propranolol $30 \mathrm{mg} / \mathrm{Kg}$ / day, which were mixed with normal laboratory powdered stock diet (containing protein $24.2 \%$, fat $5.5 \%$, sodium $0.24 \%$, and calcium $1.76 \%$ ) and were dried to be solid overnight under $40^{\circ} \mathrm{C}$. Changes of blood pressure and body weight were checked frequently and organ changes were examined microscopically at the age of 10 weeks and 15 weeks ( 5 weeks and 10 weeks after the beginning administration of anti-hypertensive drugs, respectively). Non-treated SHRs at the same age were used as controls.

Results:

1. Changes of body weight; $80-85 \mathrm{Gm}$ at 5 weeks of age, 145-152 Gm at 10 weeks, and $198-220 \mathrm{Gm}$ at 15 weeks, which show no difference between treated SHR groups and non-treated ones.

2. Changes of blood pressure; The mean of blood pressure was $105.9 \mathrm{mmHg}$ at pre-hypertensive stage, 5 weeks of age. At 10 weeks of age, significant changes were observed; blood pressure of control group raised to $151 \mathrm{mmHg}$, that of fusaric acid group was $116 \mathrm{mmHg}$, hydralazine group was $132 \mathrm{mmHg}$, and propranolol group was $135 \mathrm{mmHg}$. At 15 weeks of age, more significant changes were obtained; hypertension was completely inhibited in fusaric acid and hydralazine groups (120 $\mathrm{mmHg}$ and $132 \mathrm{mmHg}$, respectively) but hypertension was developed in propranolol group ( $160 \mathrm{mmHg}$ ) and control group (161 $\mathrm{mmHg}$ ).

3. Changes of heart and kidney weight; There was no significant difference of weight among these groups at 10 weeks or 15 weeks of age.

4. Microscopic organ changes; Organ changes are summarized on the table.

From the Second Department of Internal Medicine (Department of Cardiology, Surugadai Nihon University Hospital), and the Second Department of Pathology,* Nihon University School of Medicine, Tokyo. 


\begin{tabular}{|c|c|c|c|c|c|c|}
\hline & \multicolumn{6}{|c|}{ Kidney } \\
\hline & \multicolumn{3}{|c|}{ Interlobular Arteries } & \multicolumn{3}{|c|}{ Arcuate Artery } \\
\hline & $\begin{array}{l}\text { Perinuclear } \\
\text { Vacuolation } \\
10 \mathrm{w} \rightarrow 15 \mathrm{w}\end{array}$ & \multicolumn{2}{|c|}{$\begin{array}{c}\text { Medial } \\
\text { Thickening } \\
10 \mathrm{w} \rightarrow 15 \mathrm{w}\end{array}$} & \multicolumn{2}{|c|}{$\begin{array}{l}\text { Perinuclear } \\
\text { Vacuolation } \\
10 \mathrm{w} \rightarrow 15 \mathrm{w}\end{array}$} & $\begin{array}{c}\text { Medial } \\
\text { Thickening } \\
10 \mathrm{w} \rightarrow 15 \mathrm{w}\end{array}$ \\
\hline Control & $+\sim H \rightarrow-\sim+$ & $H$ & $\rightarrow H \sim H$ & \multicolumn{2}{|c|}{$-\rightarrow \pm$} & $-\rightarrow H \sim H$ \\
\hline Fusaric Acid & $\pm \sim+\rightarrow \pm \sim+$ & + & $\rightarrow \pm \sim+$ & $-\rightarrow$ & & $-\rightarrow \pm$ \\
\hline Hydralazine & $+\quad \rightarrow \pm \sim+$ & $\pm \sim+$ & $\rightarrow+\sim H$ & $-\rightarrow-$ & & $\pm \rightarrow \pm \sim+$ \\
\hline \multirow[t]{4}{*}{ Propranolol } & $+\quad \rightarrow-\sim \pm$ & $\pm \sim+$ & $\rightarrow \quad+$ & $-\rightarrow-$ & & $-\rightarrow+\sim H$ \\
\hline & \multicolumn{6}{|c|}{ Heart } \\
\hline & \multirow{2}{*}{\multicolumn{2}{|c|}{$\begin{array}{l}\text { Hypertrophy of } \\
\text { Cardiac Muscle } \\
10 w \rightarrow 15 w\end{array}$}} & \multicolumn{4}{|c|}{ Intra-myocardial Branch of Coronary Artery } \\
\hline & & & \multicolumn{2}{|c|}{$\begin{array}{l}\text { Perinuclear Vacuolation } \\
\qquad 10 \mathrm{w} \rightarrow 15 \mathrm{w}\end{array}$} & \multicolumn{2}{|c|}{$\begin{array}{c}\text { Medial Thickening } \\
10 \mathrm{w} \rightarrow 15 \mathrm{w}\end{array}$} \\
\hline Control & \multicolumn{2}{|c|}{$+\sim H \rightarrow+\sim H$} & \multicolumn{2}{|c|}{$\pm \rightarrow \quad+$} & \multicolumn{2}{|c|}{$-\sim+\rightarrow+$} \\
\hline Fusaric Acid & \multicolumn{2}{|l|}{$-\quad \rightarrow \quad \pm$} & \multicolumn{2}{|c|}{$+\rightarrow \pm \sim+$} & \multicolumn{2}{|c|}{$-\sim \pm \rightarrow \pm \sim+$} \\
\hline Hydralazine & \multicolumn{2}{|l|}{$+\quad \rightarrow \quad \pm$} & \multicolumn{2}{|c|}{$+\rightarrow+$} & \multicolumn{2}{|r|}{$+\quad \rightarrow \pm \sim+$} \\
\hline Propranolol & \multicolumn{2}{|c|}{$\pm \quad \rightarrow \pm \sim+$} & \multicolumn{2}{|c|}{$+\rightarrow \pm \sim+$} & \multicolumn{2}{|c|}{$\pm \sim+\rightarrow+$} \\
\hline
\end{tabular}

(Hematoxylin-Eosin staining)

Discussion:

Hypertension was completely inhibited after administration of fusaric acid and hydralazine. Fusaric acid showed stronger inhibitory effect than hydralazine. Hypertension was developed in SHR at 15 weeks of age after administration of propranolol.

Microscopically, perinuclear vacuolation and medial thickening in interlobular and arcuate arteries were observed in control (non-treated) group. And lesser perinuclear vacuolation and stronger medial thickening were also observed with aging, which means that such functional changes as vascular contraction might gradually turn into organic wall thickening.

In fusaric acid group, changes of interlobular and arcuate arteries were slight, but in hydralazine group medial thickening of interlobular arteries was observed to be moderate although hypertension was inhibited. Such humoral factors as catecholamines and renin, which were not inhibited by administration of hydralazine, could make further arteriolar changes as vasculotoxic.

In propranolol group, medial thickening of arcuate arteries was moderate, which expresses adaptation for hypertension that would be produced by relatively dominant state of $\alpha$-adrenergic receptors after administration of $\beta$-adrenergic blockers.

Microscopic changes of heart have become more remarkable in control group; hypertrophy of cardiac muscle, perinuclear vacuolation, and medial thickening of intra-myocardial branch of coronary artery. But no significant change was developed in all these treated groups. 